\title{
Development of Interrelated Voltage Regulation System for Coal Mines Energy Efficiency Improving
}

\author{
Fedor Nepsha ${ }^{1, *}$, and Roman Belyaevsky ${ }^{1}$ \\ ${ }^{1}$ T.F. Gorbachev Kuzbass State Technical University, 650000, 28 Vesennyaya St., Kemerovo, Russia
}

\begin{abstract}
In this paper, the authors propose an algorithm for interrelated voltage regulation in the power supply system of coal mine which allows to provide a normative voltage level and to minimize the level of active power consumption. A feature of the proposed algorithm is a separate consideration of discrete and nondiscrete variables. Nondiscrete variables are represented as a state matrix. The optimization of nondiscrete variables is performed for each state. The algorithm chooses a state with the minimal active power consumption. The obtained values of discrete and nondiscrete variables are transferred in the form of control signals to voltage regulation devices. In this case, the periodicity of the switching is determined by the resource of the on-load tap-changing device. The use of this algorithm will theoretically allow increasing the energy efficiency of power supply systems of coal mines.
\end{abstract}

\section{Introduction}

The task of voltage regulation in power supply systems of coal mines is the most important task, the solution of which allows to provide a normative voltage level at the terminals of electric receivers and to increase the energy efficiency of power supply systems. However the existing regulatory system does not ensure the fulfillment of these tasks. The analysis performed in [1] revealed the following features of the voltage regulation system in power supply systems of coal mines: the non-use of on-load tap-changing devices in automatic mode, the automatic control of static capacitor batteries only by the criterion of providing a standard $\operatorname{tg} \varphi$, the installation of static capacitor batteries (SCB) directly on tires of the main step-down substation, lack of coordination of voltage regulation devices. The conducted studies [1] show that in the process of functioning of the power supply systems of coal mines in normal mode the voltage deviations is about $-17 \div+7 \%$ from nominal voltage. In the after-failure mode the voltage drop reaches up to $25 \%$. Consequently the existing voltage regulation algorithm is inefficient because it doesn't assure the normative voltage. At the same time the voltage regulation system does not ensure the minimization of the level of consumption of active power at the bounds between the coal mine and the power supply organization.

${ }^{*}$ Corresponding author: nepshafs@,kuzstu.ru

*Corresponding author: nepshafs@gmail.com 
In this regard, for improving the energy efficiency of power supply systems of Kuzbass coal mines it is especially important to develop a system of interrelated voltage regulation that provides energy efficiency by optimizing the voltage level for minimizing the consumption of active power at the bounds with the power supply organization. The issue of creating a voltage regulation system in the power supply systems of coal mines was raised in [2-4]. However the system of interconnected voltage regulation in the power supply systems of coal mines has not been developed until now.

The authors consider that the system of interrelated voltage regulation in the power supply systems of coal mines should ensure the following tasks:

1. Maintaining the normative voltage level at the terminals of electric receivers;

2. Prevention of voltage increase above permissible limits in order to ensure safety of equipment;

3. Reducing the consumption of active power by adjusting the voltage level.

4. Taking into account the static load characteristics.

At the same time all voltage regulation devices must be used to regulate the voltage.

\section{Materials and Methods}

The structural diagram of the power supply system of a coal mine is shown in Fig. 1.

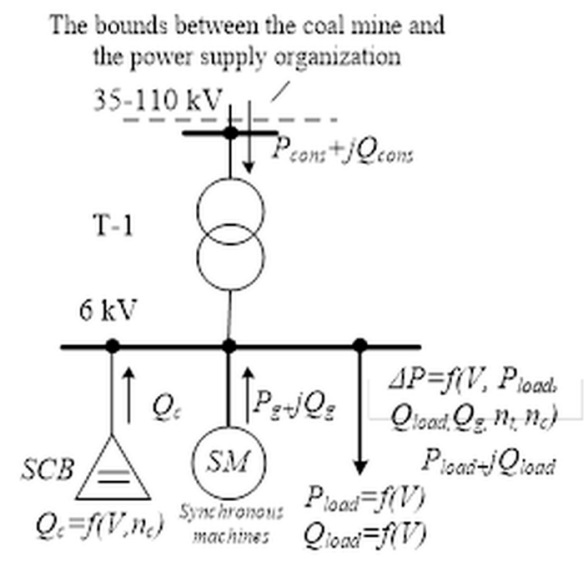

Fig. 1. Structural diagram of the power supply system of a coal mine.

To develop an algorithm for optimizing the voltage level within the power supply system shown in Figure 1, it is necessary to determine the dependent variables, boundary conditions and objective function.

Dependent variables are a set of parameters of power supply system regulating which, it is possible to change the value of the objective function. These variables can be discrete or nondiscrete. In the case of optimizing the power supply system voltage mode, the discrete variables are: the step number of the transformer on-load tap-changing device $n_{t}$, the stage number of SCB $n_{c}$. Non-discrete variables are the values of the reactive power generation by synchronous machines $Q_{g}$.

The objective function and boundary conditions for optimizing the current mode of the power supply system of a coal mine by the criterion of the minimum of active power consumption are presented in (1). 


$$
\left\{\begin{array}{l}
P_{\text {cons }}=\sum_{i=1}^{N_{1}} P_{\text {loadi }}\left(V_{i}\right)+\Delta P-\sum_{j=1}^{N_{2}} P_{g j} \\
P_{\text {cons }} \rightarrow \mathrm{min} \\
\Delta P=f\left(V, P_{\text {load }}, Q_{\text {load }}, Q_{g}, n_{t}, n_{c}\right) \\
P_{\text {load }}=f(V), Q_{\text {load }}=f(V) ; Q_{c}=f\left(V, n_{c}\right) \\
V_{\min } \leq V_{i} \leq V_{\max } \\
n_{t \cdot \min } \leq n_{t} \leq n_{t \cdot \max } \\
n_{c \cdot \min } \leq n_{c} \leq n_{c \cdot \max } \\
Q_{g \min j} \leq Q_{g j} \leq Q_{g \max j}
\end{array}\right.
$$

where $N_{1}$ is the number of load nodes; $N_{2}$ is the number of generating nodes in which $P_{g}=$ const; $V$ - voltage level at the terminals of the electric receivers; $\Delta P$ - loss of active power; $V_{\min }, V_{\max }$ - maximum and minimum voltage level limits determined by GOST requirements and passport data of electric receivers; $Q_{g m i n j}, Q_{g m a x j}$ - the maximum and minimum limitations on the level of reactive power generation determined by the passport data of synchronous machines and the $P-Q$ diagram; $P_{\text {load }}=f(V), Q_{\text {load }}=f(V)$ are the static load characteristics of coal mine electric receivers, $n_{t . m i n}, n_{t . m a x}$ is the minimum and maximum step number of the transformer on-load tap-changing device; $n_{c . m i n}, n_{c . m a x}-$ the minimum and maximum number of the stage of SCB.

\section{Results and Discussion}

Based on the analysis performed the authors developed the algorithm for optimizing the voltage level by the criterion of minimum consumption of active power in the power supply system of coal mines (Figure 2).

The algorithm assumes separate execution of simulation operations of the current mode and its optimization. This makes possible to monitor the condition of the power supply systems of coal mines and ensure maximum efficiency of the use of voltage regulation devices.

The proposed algorithm includes the execution of the following blocks:

Block 1 - preparation of initial data. It collects data on the topology of the electrical network and forms a matrix of static load characteristics.

Block 2 - update the original data and simulate the current mode. The telemetry data is collected and the initial data is corrected. According to the received data, the loads for the nominal voltage level are determined and the matrixes of nodes and branches are updated. Next the current mode is simulated. To simulate the electric regime, Newton's method with the damping coefficient determined by the Eneev-Matveyev scheme was chosen. The rationale for the chosen method for calculating the regimes is given in [7]. 


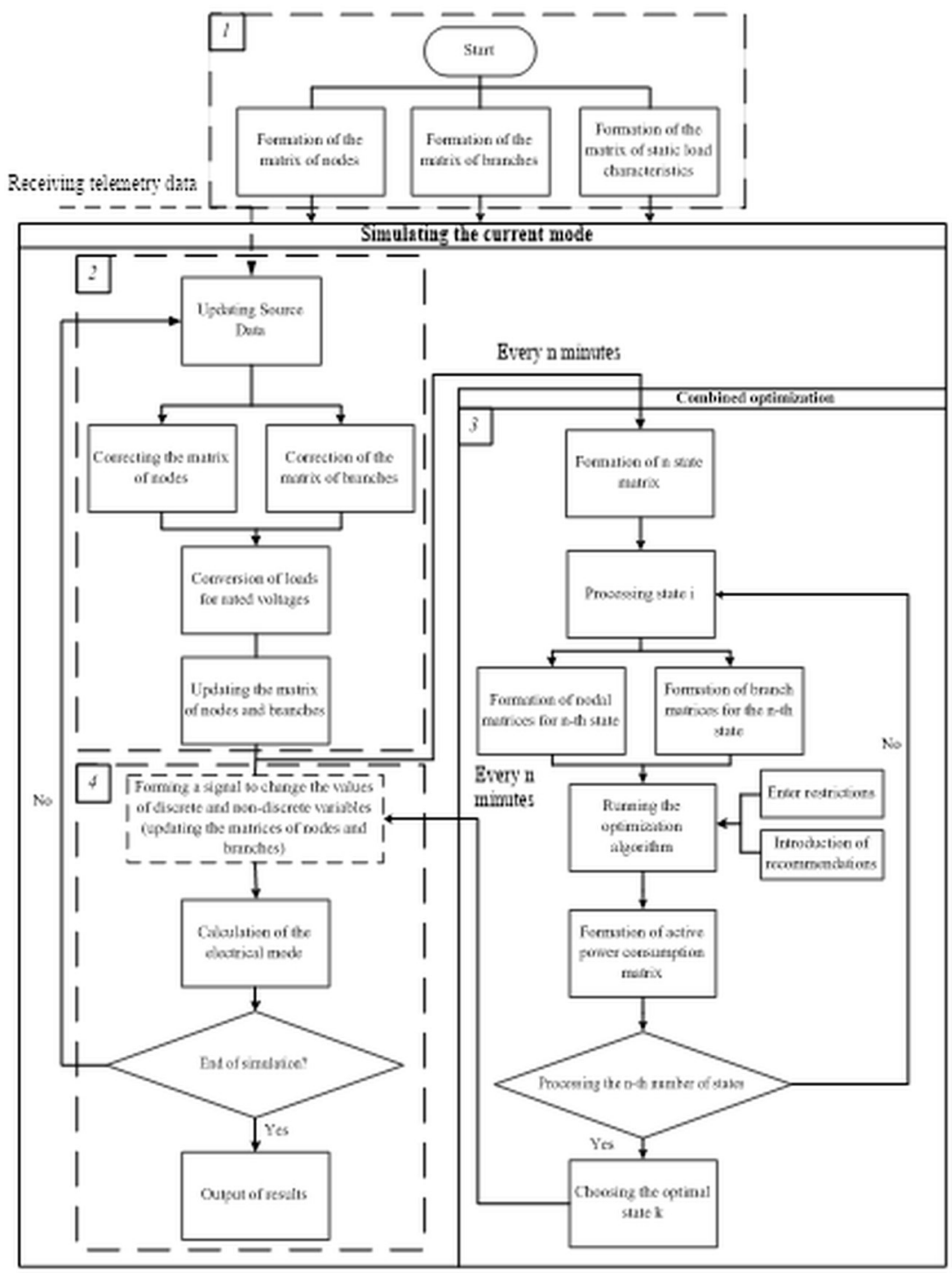

Fig. 2. General representation of the algorithm for optimizing the voltage level by the criterion of minimum consumption of active power

Block 3 - Combined optimization. The values of the dependent variables are determined in this block with definite periodicity to obtain the minimum of the objective function (1). Dependent variables that can be changed to obtain the optimal mode are shown in Table 1.

The principle of the proposed algorithm of combined optimization consists in the separate consideration of discrete and nondiscrete variables.

At the first stage of optimization, the matrix of states of the power supply systems of coal mines is formed. The constitutive state matrix makes it possible to obtain a mathematical representation of the possible states of the power supply systems of coal mines. It displays the set of all possible states of the system at the current time. 
Table 1. Types of dependent variables regulated in the process of optimizing the operating mode of power supply systems of coal mines

\begin{tabular}{|l|l|l|l|}
\hline \multicolumn{1}{|c|}{ Variable name } & Notation & Type & \multicolumn{1}{c|}{ Note } \\
\hline $\begin{array}{l}\text { Reactive power produced } \\
\text { or consumed by } \\
\text { synchronous machines }\end{array}$ & $Q_{g j}$ & $\begin{array}{l}\text { For each synchronous machine in the } \\
\text { power supply systems of a coal mine, } \\
\text { the value entering the interval } Q_{g j}= \\
{\left[Q_{g m i n j} Q_{g m a x j}\right] \text { is determined. The }} \\
\text { noper and lower limits are } \\
\text { determined by the features of each } \\
\text { synchronous machine. }\end{array}$ \\
\hline $\begin{array}{l}\text { Step number of the } \\
\text { transformer on-load tap- } \\
\text { changing device }\end{array}$ & $n_{t}$ & discrete & $\begin{array}{l}\text { For each transformer, a discrete value } \\
\text { of the variable } n_{t} \text { is found within the } \\
\text { limits } n_{t}=\left[n_{t . m i n}, n_{t . m a x}\right]\end{array}$ \\
\hline Stage number of SCB & $n_{c}$ & discrete & $\begin{array}{l}\text { Each SCB has a certain number of } \\
\text { steps. In this case, the power of the } \\
\text { SCB is equal to the discrete value } \\
\text { from the series } n_{c}=\left[n_{c . m i n}, n_{c . m a x}\right] .\end{array}$ \\
\hline
\end{tabular}

Each row of the matrix represents the possible state of the system and each column represents the number of possible variations of the individual variable. The number of columns in the matrix corresponds to the number of discrete voltage regulators and the number of lines corresponds to the possible number of discrete states of the system. The number of possible number of discrete states of the system directly depends on the number of discrete voltage level regulators. The number of possible states is very important, because it directly affects the speed of calculations. For example, if two power transformers with the number of steps of the transformer on-load tap-changing device 19 are installed at the main step-down substation and two SCB devices with 3 control stages are installed on the tires of the main step-down substation, the number of possible system states will be $19^{2}$ - $3^{2}=3249$. Conditionally assuming that the optimization time of the objective function for nondiscrete variables is 1 second, the time required for the analysis of all possible states can take a long time. Therefore, it is necessary to reduce the number of possible combinations.

One way to reduce the possible number of combinations is to separately consider the networks fed from each transformer of the main step-down substation. This method is quite acceptable because in the normal mode of the electrical network, the electric receivers, powered from each transformer, can be considered independent. Thus, when considering radial networks, it becomes possible to reduce the number of possible combinations of discrete variables.

The second way is that the transformer on-load tap-changing device can not immediately move from the current state to the extreme state. In other words, the transformer on-load tap-changing device switches the step number in sequence. Switching time is from 3 to 10 seconds. In this case, irrespective of the switching duration, the on-load tap-changing device have a lock that allows the selector to pass only one step. Therefore, it is not meaningful to consider a state matrix with the number of possible positions more than 3 . Thus, 3 positions of the transformer on-load tap-changing device are considered: $\mathrm{n}_{\text {cur }}$ (the current step number), $\mathrm{n}_{\text {cur }+1}, \mathrm{n}_{\text {cur- } 1}$. Taking into account the two above hypotheses, the number of possible states is reduced to $3^{1} \cdot 3=9$.

The second stage consists in recognizing the matrix of states and performing optimization for nondiscrete parameters. At each stage of the search for the objective function, a call to the matrix of states is performed.

To solve the problem of nonlinear conditional optimization, the authors propose the method of sequential quadratic programming (SQP) and the internal point method. According to [8], these methods are the most powerfull for such problems. It is suggested in 
the practical implementation of the developed algorithm to consider these optimization methods and compare them by two indicators:

1. The effectiveness of the optimization algorithm which is estimated by the amount of power reduction consumed from the supply network $\Delta P_{\text {cons }}(\mathrm{kW})$.

2. The speed of the algorithm (ms). The optimization process should be carried out in real time with a specified periodicity. Therefore, to exclude decision-making with delay, it is important that the implementation of the algorithm be as fast as possible.

After optimization of each possible states of the power supply systems of coal mines included in the state matrix. The state with the lowest value of the consumed active power is selected from the considered states.

Block 4 - Block for generating control signals for changing values of dependent variables. After optimization, matrix and node matrices are corrected, and a signal is generated for changing the dependent variables. Then the operation of optimizing the dependent variables is repeated at the specified periodicity.

The frequency of the optimization of dependent parameters is determined by the switching resource of the transformer on-load tap-changing device.

\section{Conclusions}

1. On the basis of the studies carried out, the authors developed the algorithm for optimizing the voltage level by the criterion of minimum consumption of active power in power supply systems of coal mines.

2. The proposed algorithm takes into account the static load characteristics which increases its accuracy and reliability and allows the use of electric receivers to regulate the consumption of active and reactive power.

3. The developed algorithm allows to take into account the work of reactive power compensation devices if they have a discrete control range.

4. It is proposed to perform joint optimization of discrete and nondiscrete variables by forming state matrices. To accelerate the calculations in the formation of the state matrix with respect to the transformer on-load tap-changing device, it is proposed to take into account only the neighboring positions of the transformer on-load tap-changing device.

To further approbation of the developed algorithm, it is necessary to implement the developed algorithm in the software environment and apply it in relation to the imitating model of the power supply system of a coal mine.

\section{References}

1. F. S. Nepsha, V. M. Efremenko, Industrial power engineering, 11, (2017)

2. Yu. P. Minovskiy, Electricity efficiency of coal mine faces (Nedra, Moscow, 1990)

3. E. A. Konyukhova, Regulation of power consumption of an industrial enterprise with an interconnected choice of voltage mode and reactive power compensation. (Nedra, Moskow, 1998)

4. V. V. Polishchuk, Regulation of the voltage regime in the distribution networks of 6 (10) kV mining enterprises. (SPMI, St. Petersburg, 1996)

5. D. M. Tarasov, Management of a mode of voltage of territorially dispersed electric receivers of the mountain enterprises. (SPMI, St. Petersburg, 2003)

6. A. P. Shevchuk, Increase of efficiency of group regulation of voltage in distributive networks of industrial enterprises in the conditions of territorially distributed electric consumers. (SPMI, St. Petersburg, 2014) 
7. J. Nocedal, S. J. Wright, Numerical Optimization (Eds. Springer, New York, 2006) 